\title{
Development and implementation of an automatic system for verification, validation and delivery of laboratory test results
}

\author{
Gian Cesare Guidi', Giovanni Poli' ${ }^{1}$, Antonella \\ Bassi ${ }^{1}$, Luca Giobelli ${ }^{2}$, Pier Paolo Benetollo ${ }^{3}$ \\ and Giuseppe Lippi ${ }^{1, *}$ \\ ${ }^{1}$ Laboratorio di Analisi Chimico-Cliniche ed \\ Ematologiche, Azienda Ospedaliera di Verona, \\ Policlinico G.B. Rossi, Verona, Dipartimento di \\ Scienze Morfologico-Biomediche, Università di \\ Verona, Verona, Italy \\ ${ }^{2}$ Servizio Sistemi Informativi, Azienda Ospedaliera \\ di Verona, Verona, Italy \\ ${ }^{3}$ Direzione Sanitaria, Azienda Ospedaliera di Verona, \\ Verona, Italy
}

\begin{abstract}
Background: The verification/validation of laboratory test results is one of the most critical aspects of the total testing process, which may produce conflicts between competencies and duties at the point of professional crossroads. This process has centered for decades on the human component, with positive effects as well as potential adverse consequences (postanalytical errors). Manual validation of data is a time-consuming activity, is inherently subjective and arbitrary, and requires the constant presence of postgraduate physicians or biologists within the laboratory with adverse economical and organizational impacts. To overcome these inherent limitations, we have developed and implemented in our stat department an automatic system for verification, validation and delivery of laboratory results.

Methods: The procedure is based on automatic validation of test results by an expert system, coupled with remote wireless connection, which allows the laboratory professional "on call" to access, visualize, analyze, validate and deliver alert values (suspect, erroneous or critical) using a small laptop. This system also provides five phases where preanalytical and analytical errors can be identified and handled. Results and conclusions: Six months following implementation of this innovative system, which can be customized to facilitate a wide variety of laboratory workflow models, the reporting efficiency of our stat laboratory has greatly improved, reducing manual
\end{abstract}

*Corresponding author: Prof. Giuseppe Lippi, Sezione di Chimica Clinica, Dipartimento di Scienze MorfologicoBiomediche, Ospedale Policlinico G.B. Rossi, Piazzale Scuro, 10, 37134 Verona, Italy Phone: +39-045-8124516, Fax: +39-045-8027484,

E-mail: giuseppe.lippi@univr.it; ulippi@tin.it Received July 4, 2009; accepted July 30, 2009; previously published online October 12, 2009 data entry, and increasing the timeliness and utility of test results.

Clin Chem Lab Med 2009;47:1355-60.

Keywords: information technology; laboratory testing; results; validation; verification.

\section{Introduction}

The widespread diffusion of technologies, driven in large part by information technology (IT) has paved the way for substantial advances and innovations in clinical laboratory sciences (1-3). A technology- and analytically-oriented approach, focused primarily on producing test results with the most productive and effective equipment, has been gradually replaced with a new patient-centered perspective. With this new perspective, awareness of the clinical significance of laboratory test results has become the core of the total testing process (1-3). The primary goal of laboratory testing is the generation of reliable results that accurately reflect the physiological or pathological state of the patient. As such, they can be useful for clinical decision-making and impact patient health and safety. However, any condition (or error) that interferes with the clinical usefulness must be considered disadvantageous, if not harmful to the patient (4-6). Such a deduction is supported by two considerations.

The former consideration must be considered with respect to the concept of total quality in laboratory testing. The ability to track several steps of the total testing process, from sample collection to reporting of results, is indeed a major responsibility for the laboratory staff, but this process has also produced a broadening of responsibilities in the daily practice $(7$, 8 ). The progressive decline in analytical bias and uncertainty in several areas of testing, such as the diagnosis of acute myocardial infarction (9), has greatly enhanced the role and the responsibility of laboratory professionals for unmasking uncertainties and identifying errors outside the analytical phase. These uncertainties and errors can jeopardize the efficiency and the efficacy of the global testing process.

The latter consideration, which is a direct consequence of the former, arises from the wide spectrum of professional figures working in the clinical laboratory. These include laboratory technicians (LT), medical doctors (MD), and other medical laboratory technologists (MLT). While there is a certain degree of heterogeneity among countries and even within 
national laboratories, each professional figure has specific competencies and responsibilities for guaranteeing that test results are correct, and thereby useful for clinical decision-making.

One of the most critical aspects of the total testing process which may lead to conflicts between competencies and duties is the verification/validation of the test results $(10,11)$. The verification/validation process is crucial as it allows for the identification of a variety of errors occurring in the preanalytical and analytical phases. The verification/validation process prevents the inadvertent notification of suspect or erroneous results to the wards. It also allows the identification of bottlenecks or flaws in the entire testing process and helps open discussion of critical situations with clinicians. A deeper analysis of unexpected (either critical or erroneous) results may include checking of sample integrity and identity, reanalysis of samples, as well as complete revision of the testing process to identify potential critical issues and vulnerabilities. With respect to the clinic-laboratory interface, the timely notification of results, the inclusion of interpretative comments with laboratory reports, and clinical discussion of cases are important aspects that help prevent potential harm to the patient due to delivery of incorrect test results (11). Last but not least, verification and validation of test results allows strong reaffirmation of the role of consultant that laboratory professionals should assiduously pursue in their profession.

The verification and validation of laboratory results has been centered for decades on the human component. This has positive effects, but also has potential adverse consequences (postanalytical errors) (12, 13). In many clinical laboratories, routine and stat samples follow the same path, and are processed simultaneously on the same instruments that inevitably requires the presence of a full-time technical staff. The validation of test results is a time-consuming activity, which is complicated by the shortage of staff currently affecting healthcare systems, and particularly clinical laboratories. Other critical aspects in the process of manual validation is the degree of subjectivity, and the tediousness of the process due to the need to check each single result before it can be delivered to caregivers. To overcome the inherent limitations of manual validation, some computer programs have been developed recently. In the field of laboratory medicine, these programs consist primarily of a set of rules dealing with test results (14-18). They produce various types of flags for pathological values, abnormal deviations in the longitudinal comparison of test results, results that are inconsistent with certain patient characteristics, and incongruity with other test results. These rules are derived from the knowledge of a body of experts which has been translated into rules used to review huge amounts of data (test results), and always with the same objective approach. The expert systems can be configured according to the facility and staff, and can be modified according to local experience and expertise. The purpose of this article is to present the results of imple- mentation in the stat department of an automated system for verification, validation and delivery of laboratory results.

\section{Materials and methods}

\section{Description of the system}

The described system was developed in cooperation with Metafora Informatica (Milan, Italy) at the Clinical Chemistry Laboratory of the Verona Academic Hospital. Some specific components of the system are described in Table 1. The information flow is described in Figure 1. Samples are first checked for integrity, and possible preanalytical problems are recorded according to a standardized protocol described elsewhere (19). Suitable samples are processed immediately, raw data are checked by a LT directly on the instrument, with particular focus on the presence of flags for analytical errors or interferences (established on the basis of the serum indices). The data are transmitted from the analyzer to a computer laboratory that serves as a concentrator and which finally retransmits the data to the laboratory information system (LIS) located on the main server in the Informatics Department of the Hospital. Before data can be entered into the LIS, a second check of validity is performed (Figure 1), because the LIS is configured with a pre-defined list of criteria for acceptability (defined essentially on the basis of the lower and upper limits of linearity for each test). When test results lie outside these limits or contain flags produced by the instrument (e.g., sample redilution because results were outside the limit of linearity of the method, abnormal kinetics, etc.), they are not automatically entered into the LIS but instead must be entered manually by the operator LT. This represents a second defence since all the data that contain various types of flag must be checked in advance by the staff before they can be entered into the LIS. At this phase of the process, data can be displayed on laboratory computers with the software Concerto (Metafora Informatica) and eventually released. However, before proceeding with the "analytical validation", the technical staff has an additional validation tool in Concerto which is the longitudinal comparison of the new data with previous testing results from the same patient (if available). Results might be flagged when: (a) values are critical, according to objective criteria from established guidelines and scientific recommendations, (b) the deviation from previous results (delta check) exceeds the limit of biological plausibility, (c) there is a violation of congruence when a given parameter is compared with others (e.g., a normal value of body urea nitrogen in the presence of increased creatinine concentration, or vice versa). The analytical validation makes the data available to the expert system for verification and validation (Validation Assistée pour laboratoire, $V A L A B)$. VALAB is located on a dedicated server in the Infor-

Table 1 Specific components of the system.

Hardware
- Main server, located in the Informatic System
Department of the Hospital
- Computer in the laboratory
- Three laptops with wireless connection
Software
- Laboratory Information System (Concerto, Metafora)
- Expert system for validation of test results (VALAB)
- Remote connection system (VPN Cisco)
- System of remote computer emulation (CA Unicenter
remote controller)




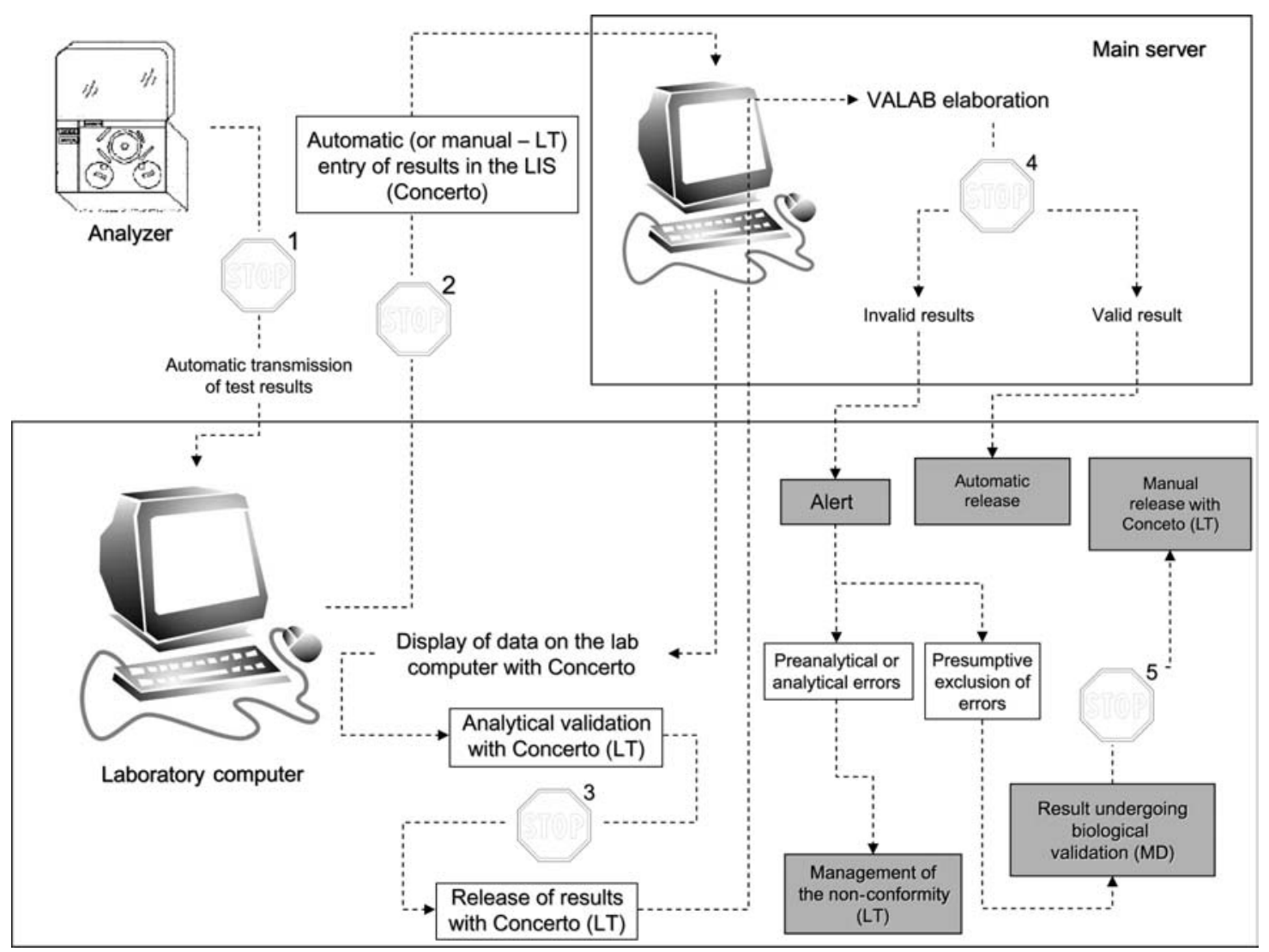

Figure 1 Information workflow.

LIS, laboratory information system; LT, laboratory technician; MD, medical doctor.

matics Department of the Hospital and operates using a series of algorithms (described in detail elsewhere) (14-17) based on: (a) comparison of results with critical values, (b) biological plausibility of the data according to the previous, (c) decision-making algorithms that compare results of multiple parameters to further verify their biological plausibility, (d) requesting department and type of the request (routine or stat). When results do not violate any of the above-mentioned rules, they are automatically validated, released and delivered to the requesting physicians, who can display the results on a dedicated computer in the ward. Conversely, when results do not meet one or more of the above-mentioned criteria, the software sends an automatic alarm message ("alert") on a computer located in the stat department (Figure 2). The technical staff can identify the reason for the alert generated by the software Concerto (e.g., critical values, incongruity with other tests, variation exceeding the biological limit of variability). If the cause can be reliably determined to be a preanalytical or analytical error, the technical staff records the problem and handles the specimen as specified in the quality manual of our laboratory. However, when the reason generating the alert cannot be attributed to an error, the technical staff immediately notifies the MD of the situation, who may be available in the stat department, or on call outside the laboratory.

The system developed in our laboratory has specific characteristics when compared with similar solutions adopted at other facilities (Figure 3). The organization of the laboratory relies on the active presence of a MD between 08:00 and 20:00 on weekdays, and between 08:00 and 13:00 during holidays. When the MD is on call and is outside the laboratory, he/she is equipped with a laptop. The laptop, system software operates with Windows XP and includes a program that enables connection from locations remote to the hospital network (Cisco VPN Client, Cisco Systems Inc, San Jose, CA, USA). This software allows for a secure connection and protects the network from unauthorized access. The laptop operates using a wireless internet connection (Alice Mobile USB Pen Drive Card, Telecom, Italy) and a dedicated control program (CA Unicenter Remote Controller, Computer Associates, Islandia, NY, USA) that can emulate a computer in the laboratory. The computer that is emulated is usually the one where the automatic "alert" generated by VALAB is displayed. Using a remote connection to a computer in the stat department, the MD on call can identify the cause of the alert, examine other data, review previous results from the patient, and then proceed with the validation or, if necessary, contact the doctor who is in charge of the patient and discuss the case (Figure 2). Statistics showing the daily average [ \pm standard deviation (SD)] of the functionality of this system during the first 6 months following implementation are shown in Table 2.

\section{Results and discussion}

Like several other phases of the total testing process, validation of laboratory results has derived great benefits from advancements in computer technology. The final outcome of validation is the production of the laboratory report. The significance of laboratory data is becoming increasingly clear for clinical decisionmaking when one considers that nearly $2 / 3$ of clinical decisions are more influenced by laboratory data (20). The laboratory report should be considered for its 


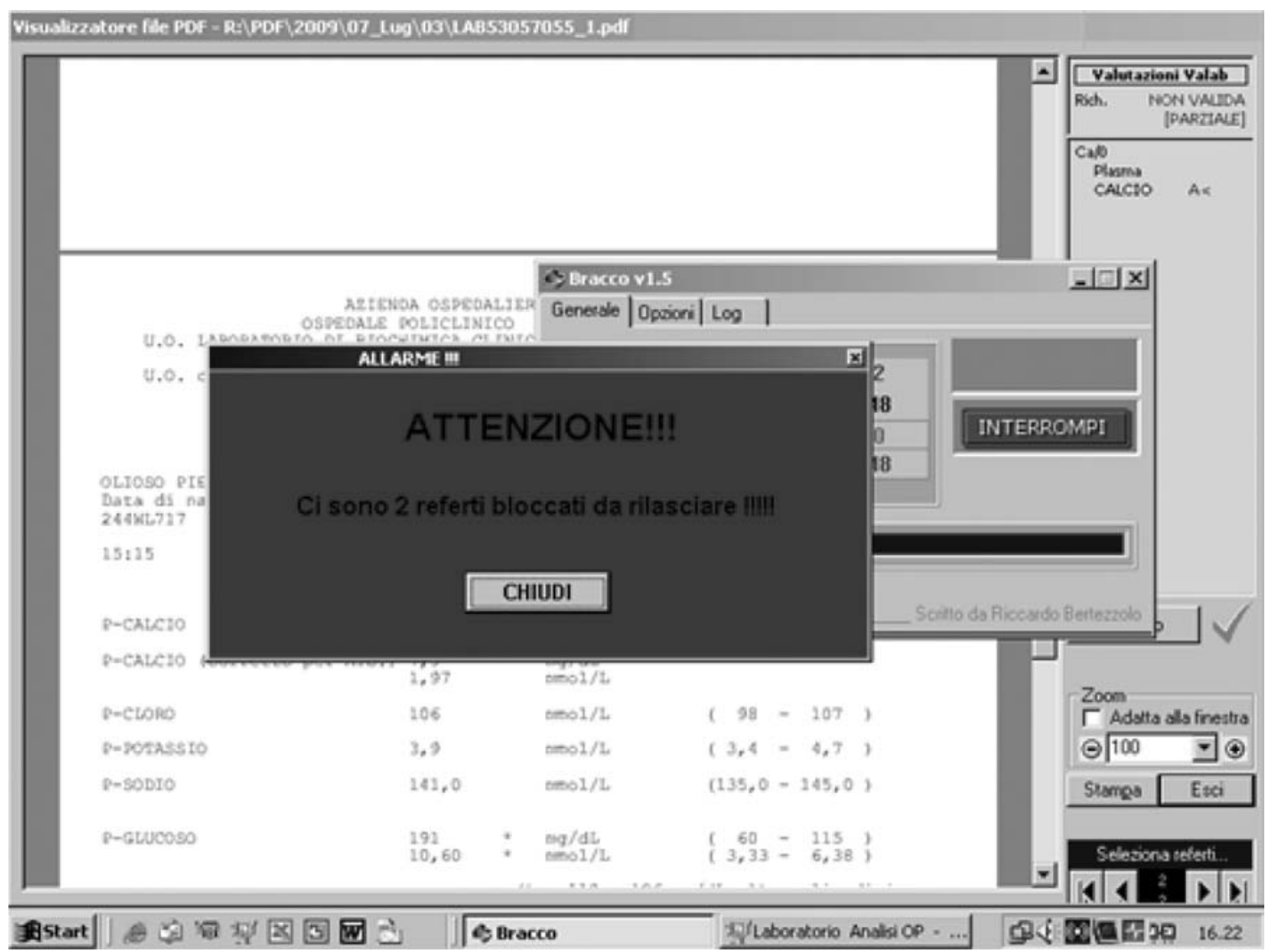

Figure 2 Alert appearing on the laboratory computer and on the remote laptop of the medical doctor on call. Translation: ATTENTION!!! There are two results to be released!!!!!

semeiological value; the presence of reference intervals (ever more individualized and longitudinal) (8), comments, notes and charts, enables it to undergo a transformation from a mere list of numerical results, to the genuine expression of reasoning of the MD and MLT who operate with the valuable and irreplaceable aid of LTs. According to current regulations and taking into account the professional profile of LTs in most countries, their activities are performed independently with the technical and professional assignment of responsibility for specific procedures relegated primarily to preanalytical and analytical issues (21, 22). The LT provides a substantial contribution to total quality management of laboratory diagnostics. They identify potential preanalytical problems, analytical errors and analytical interferences. There is a specific area where LTs can express their technical competence with the analytical validation. This area is clearly distinct from biological validation/verification, which is under the responsibility of the MD and MLT, and is required by organizations and legislation in some countries. Analytical validation warrants adherence to, and fulfilment of, procedures and standards that together contribute to the accuracy of the analytical process. However, it also provides essential elements of traceability of the process so that any abnormality, error or deviation from the rules can be identified and recorded as a "non-conformity".

Through validation of test results, laboratory professionals can demonstrate their professionalism, providing a global overview of the different phases of the diagnostic process. This overview spans the collection and handling of biological samples (preanalytical phase), to analysis (analytical phase) and delivery of reliable results (post analytical phase). Due to the growing complexity and specialization of laboratory diagnostics, the validation process is becoming a critical step. This process requires a high degree of knowledge, time and commitment, especially when operating under manual validation because any individual report must be browsed, sequentially. The limits of manual validation have already been mentioned. Thus, the contribution of automation and IT may be crucial to rationalize and standardize (or harmonize) the preanalytical and analytical processes as well as the postanalytical phase. The system developed in our laboratory and supported by IT has considerable clinical and organizational advantages. Each model of efficient clinical governance is based on a system of defensive barriers. These barriers should be redundant and multifaceted so that the probability that an accident (error) turns into an accident (adverse consequence for the patient) can be minimized $(5,23)$. In compliance with these requirements, we have implemented multiple steps for verifying the analytical and clinical reliability of results. These are articulated using different levels of alarms (instrumental check, analytical acceptability, biological plausibility, clinical reliability) for increasing the possibility of identifying and blocking block errors (Figure 1). The 


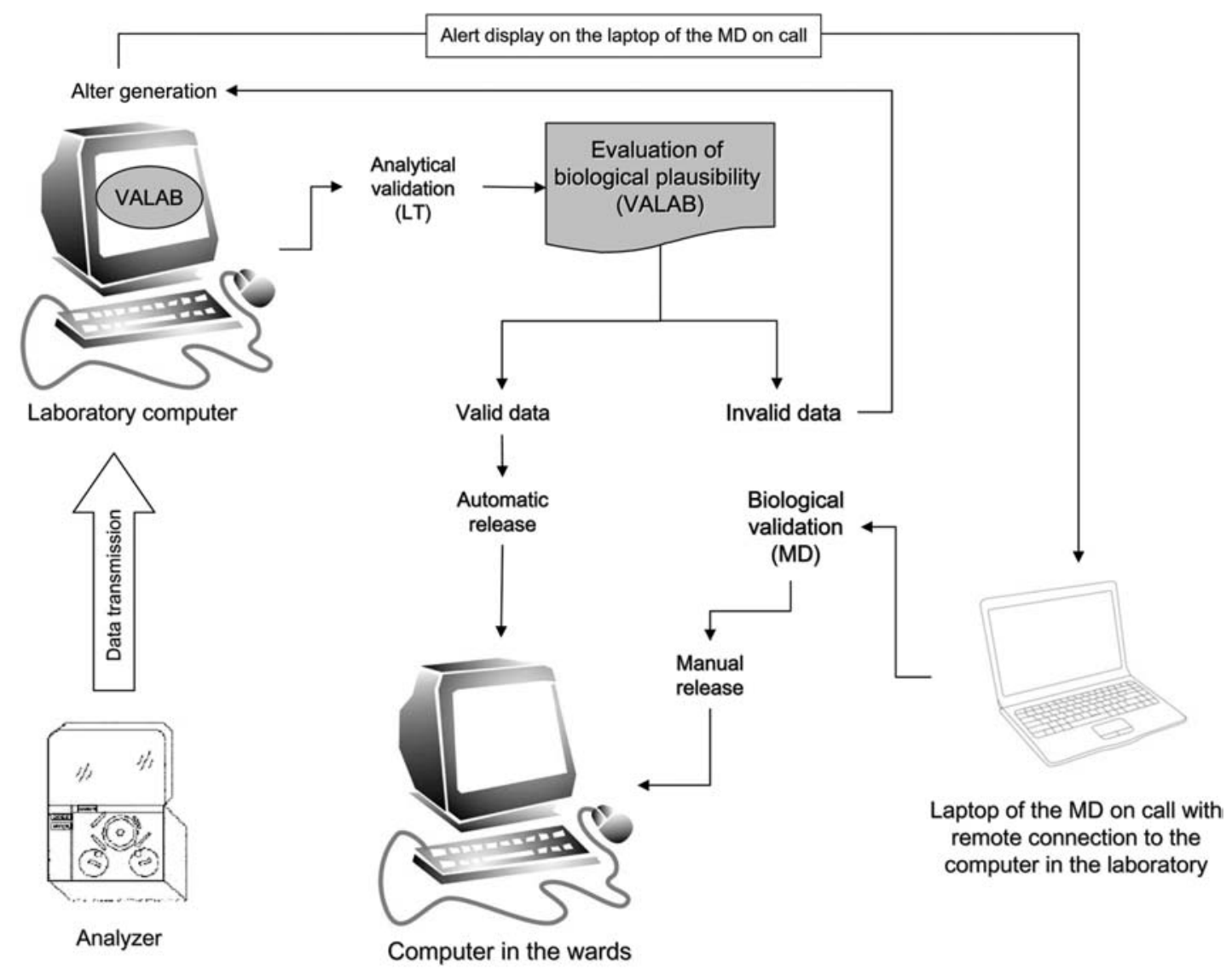

Figure 3 Process of remote validation by the medical doctor on call.

LT, laboratory technician; MD, medical doctor.

Table 2 Daily average ( \pm standard deviation) functionality of the system during the first 6 months following implementation.

\begin{tabular}{llr}
\hline & Number & Percentage, $\%$ \\
\hline Laboratory requests (stat) & $161 \pm 13$ & 100 \\
Laboratory requests (stat) containing one or more alerts & $13 \pm 3$ & 8.1 \\
$\quad$ Laboratory requests (stat) validated by the MD on call & $12 \pm 3$ & 7.5 \\
$\quad \begin{array}{l}\text { Laboratory requests (stat) non-validated (for the presence } \\
\text { of preanalytical or analytical errors) }\end{array}$ & $1 \pm 0$ & 0.6 \\
Direct notifications of dubious/critical values by the MD on call & $2 \pm 1$ & 1.2 \\
\hline Mean time elapsed from analytical LT and biological MD validation & $4 \pm 2$ min \\
\hline
\end{tabular}

$\mathrm{LT}$, laboratory technician; MD, medical doctor.

average time between the analytical LT and biological $M D$ validation has been associated with a negligible increase in the turn around time $(4 \pm 2 \mathrm{~min})$, which is offset by the greater degree of control over the quality of the data. The second advantage of the procedure is organizational. Using a remote connection, the MD on call can get connected at any time of the day to a computer in the laboratory and access a wide range of information (analytical alarms or biological plausibility). The MD is able to visualize, troubleshoot and validate any single report that has generated an alarm by the expert system (Figure 3). This solution does not require the establishment of a service with the active presence of MDs in the laboratory, since they can connect from anywhere to the LIS. This results in favorable economic and organizational revenues for the laboratory and hospital administration. The increasing availability of computing devices, such as smart phones or laptops provides further important developments because laboratory professionals on call can connect remotely without needing bulky hardware. The other advantage of implementing an expert system for validating laboratory results is the ability to overcome the subjectivity and arbitrary nature associated with clinical judgement. The rules used in VALAB are determined on the basis of guidelines, recommendations and scientific publications.

Postanalytical activity has two main goals for improving patient safety: result interpretation and communication of critical values (24). Both are critical issues and their success requires cooperation between the great advances in IT and other technological advances that are revolutionizing laboratory activities. It was recently shown that automated communication of test results improves the timeliness of notification, prevents potential errors, improves the 
likelihood of reaching the physician on call, and provides important decision support (25). Six months following implementation of this innovative system, which can also be customized to facilitate a wide variety of laboratory workflow models, we have also shown that the reporting efficiency of our stat laboratory has greatly improved, reducing manual data entry, and increasing the timeliness and utility of test results.

\section{Acknowledgements}

The authors acknowledge Ing. Riccardo Bertezzolo for his collaboration in developing the system.

\section{References}

1. Panteghini M. The future of laboratory medicine: understanding the new pressures. Clin Biochem Rev 2004;25: 207-15.

2. Guidi GC, Lippi G. Laboratory medicine in the 2000s: programmed death or rebirth? Clin Chem Lab Med 2006; 44913-7.

3. Guidi GC, Lippi G. Will "personalized medicine" need personalized laboratory approach? Clin Chim Acta 2009; 400:25-9.

4. Plebani M. Exploring the iceberg of errors in laboratory medicine. Clin Chim Acta 2009;404:16-23.

5. Lippi G, Guidi GC. Risk management in the preanalytical phase of laboratory testing. Clin Chem Lab Med 2007;45: 720-7.

6. Lippi G, Fostini R, Guidi GC. Quality improvement in laboratory medicine: extra-analytical issues. Clin Lab Med 2008;28:285-94.

7. Panteghini M. Traceability, reference systems and result comparability. Clin Biochem Rev 2007;28:97-104.

8. Guidi GC, Lippi G, Solero GP, Poli G, Plebani M. Managing transferability of laboratory data. Clin Chim Acta 2006:374:57-62.

9. Dolci A, Panteghini M. The exciting story of cardiac biomarkers: from retrospective detection to gold diagnostic standard for acute myocardial infarction and more. Clin Chim Acta 2006;369:179-87.

10. Ricós C, García-Victoria M, de la Fuente B. Quality indicators and specifications for the extra-analytical phases in clinical laboratory management. Clin Chem Lab Med 2004;42:578-82.

11. Plebani M. Interpretative commenting: a tool for improving the laboratory-clinical interface. Clin Chim Acta 2009;404:46-51.

12. Plebani M, Carraro P. Mistakes in a stat laboratory: types and frequency. Clin Chem 1997;43:1348-51.

13. Witte DL, VanNess SA, Angstadt DS, Pennell BJ. Errors, mistakes, blunders, outliers, or unacceptable results: how many? Clin Chem 1997;43:1352-6.

14. Valdiguié PM, Rogari E, Philippe H. VALAB: expert system for validation of biochemical data. Clin Chem 1992; 38:83-7.

15. Valdiguié PM, Rogari E, Corberand JX, Boneu B. The performance of the knowledge-based system VALAB revisited: an evaluation after five years. Eur J Clin Chem Clin Biochem 1996;34:371-6.

16. Fuentes-Arderiu X, Castiñeiras-Lacambra MJ, PanaderoGarcía MT. Evaluation of the VALAB expert system. Eur J Clin Chem Clin Biochem 1997;35:711-4.

17. Marchand M, Guibourdenche J, Saada J, Le Men H, Porquet $D$, Demelier JF. Real time validation of paediatric biochemical reports using the Valab-Biochem system. Ann Clin Biochem 1997;34:389-95.

18. Oosterhuis WP, Ulenkate HJ, Goldschmidt HM. Evaluation of LabRespond, a new automated validation system for clinical laboratory test results. Clin Chem 2000;46: 1811-7.

19. Lippi G, Banfi G, Buttarello M, Ceriotti F, Daves M, Dolci $A$, et al. Recommendations for detection and management of unsuitable samples in clinical laboratories. Clin Chem Lab Med 2007;45:728-36.

20. Plebani M. Charting the course of medical laboratories in a changing environment. Clin Chim Acta 2002;319:87100.

21. Guidi GC, Lippi G. Undergraduate education in Laboratory Medicine. Clin Chim Acta 2008;393:9-12.

22. Guidi GC, Lippi G, Plebani M. The holistic approach to teach laboratory medicine. Clin Chim Acta 2008;393:513.

23. Lippi G. Governance of preanalytical variability: travelling the right path to the bright side of the moon? Clin Chim Acta 2009;404:32-6.

24. Piva E, Plebani M. Interpretative reports and critical values. Clin Chim Acta 2009;404:52-8.

25. Piva E, Sciacovelli L, Zaninotto M, Laposata M, Plebani M. Evaluation of effectiveness of a computerized notification system for reporting critical values. Am J Clin Pathol 2009;131:432-41. 